Archive for

Organic Chemistry
Arkivoc 2018, part vii, 0-0

to be inserted by editorial office

\title{
Synthesis and antimicrobial activity of some new of 2-(furan-2-yl)-1- (piperidin-4-yl)-1H-benzo[d]imidazole derivatives
}

\author{
Tejasvi H. Parmar, ${ }^{a}$ Chetan B. Sangani, ${ }^{* a}$ Nilesh D. Parmar, ${ }^{b}$ and Pradip C. Bhalodiya ${ }^{a}$ \\ ${ }^{a}$ Department of Chemistry, Shri M.M Patel Institute of Sciences and Research, Kadi Sarva Vishwavidyalaya, \\ Gandhinagar - 382023, India \\ ${ }^{b}$ Department of Chemistry, Saurashtra University, Rajkot-360 005, Gujarat, India \\ Email: chetansangani1986@yahoo.com; tejashparmar@gmail.com
}

Received 10-24-2018

Accepted 12-09-2018

Published on line $12-27-2018$

\section{Abstract}

We report a series of new heterocyclic compounds containing the imidazole scaffold were synthesized such as 2-(furan-2-yl)-1-(piperidine-4-yl)-1H-benzo[d]imidazole derivative. Due to the biological activities of imidazole as antimicrobial agents, in the present work, all the synthesized compounds were characterized by ${ }^{1} \mathrm{H}$ NMR and LC-MS analysis and some of the compounds are characterized by ${ }^{13} \mathrm{C} \mathrm{NMR}$. All the synthesized compounds were evaluated for their antimicrobial activity against Gram +ve and Gram -ve bacteria and different fungal species which demonstrated good to moderate antimicrobial activity, in which compounds $7 \mathbf{b}$ and $7 \mathbf{l}$ shows highest antimicrobial activity.

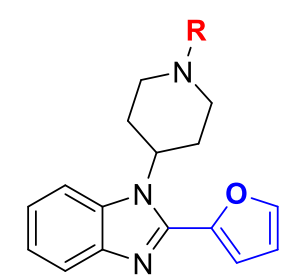

$\mathrm{R}=$ Different acyl chloride, acids and sulphonyl chloride derivatives

Keywords: Sodium dithionate, HATU reagent, furan-2-carbaldehyde, antimicrobial activity 


\section{Introduction}

Heterocycles of benzimidazole are important substructures identified in natural products and pharmacologically active molecules. ${ }^{1-5}$ A different derivative of imidazole act as an inhibitor of p38 MAP kinase $^{6}{ }^{6}$ glucagon receptors, ${ }^{7}$ plant growth regulators, ${ }^{8}$ therapeutic agents, ${ }^{9}$ antibacterial, ${ }^{10}$ and also antitumor, ${ }^{11}$ some of the derivatives used as an ionic liquid which highly benefits to the green chemistry. The synthesis of benzimidazole derivatives plays important role in the biological activities of these compounds induced by the heterocyclic ring. Benzimidazole derivatives display a wide range of biological and pharmaceutical active such as antiulcer activity antimicrobial, antiviral, antidiabetic and anticancer activity. ${ }^{12-17}$

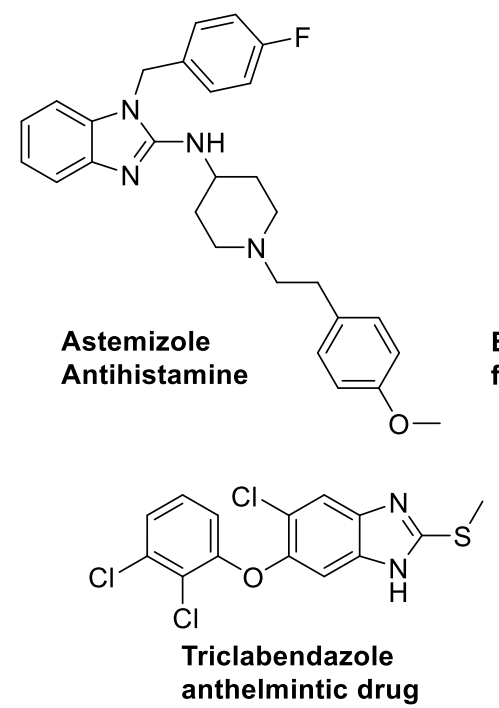

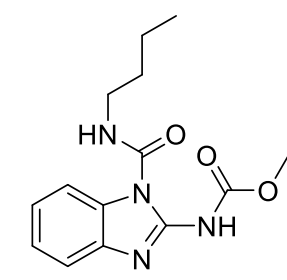

Benomyl (Benlate) is a fungicide

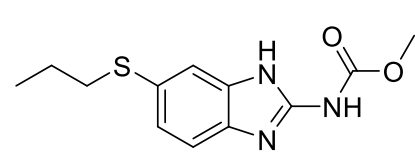

Albendazole anthelmintic drug<smiles>COC(=O)Nc1nc2ccc(C(=O)c3ccccc3)cc2[nH]1</smiles>

Mebendazole anthelmintic drug

Figure 1: Representative examples of some drugs containing a benzimidazole moiety

Commonly benzimidazole synthesized by condensation with carboxylic acids or their functional derivatives and $o$-aminoanilines. ${ }^{18-24}$ Drawbacks of these conditions are dehydrating reaction conditions, high temperature, and very strong acid catalysts.

Intramolecular cyclization of o-bromoaryl derivatives in the presence of copper(II)oxide nanoparticles in solvent DMSO, ${ }^{25}$ and the intramolecular cyclocondensation of some aryl amino oximes by using of methane sulfonyl chloride and triethylamine ${ }^{26}$ can also be used to produce benzimidazole derivatives. However, all these synthetic routes require expensive metal catalysts, toxic solvents.

Due to diverse range of biological activity and use of benzimidazole derivative in material chemistry and use as ionic solvent development of new synthetic method is an important area. We have synthesized novel benzimidazole derivatives in four steps via reductive cyclization of $n$-piperidine substituted nitroaniline with 2-furfuryl using sodium dithionite ${ }^{27,28}$ and piperidine amine coupling with commercially available acid, acyl chloride, and sulphonyl chloride.

\section{Results and Discussion}

In this work we describe the synthesis of amide and sulphonamide derivative of 2-(furan-2-yl)-1-(piperidine-4$\mathrm{yl})-1 H$-benzo[d]imidazole from $\mathrm{n}$-substituted benzene diamine. Spectral data and physical data of this 
molecules are not reported in the literature. For these molecules starting material are commercially available and reaction conditions having good yield, no hazardous chemicals and less reaction time.

1-Fluoro-2-nitrobenzene and tert-butyl 4-aminopiperidine-1-carboxylate coupling reaction by using ACN, TEA (3 eq) at $70{ }^{\circ} \mathrm{C}$ for overnight with $70 \%$ yield. For this coupling reaction performed at gram scale, product 3 was isolated pure without column purification and directly used in the next step with furan-2 aldehyde (4) in the presence of dithionite (4 eq.), $\mathrm{MeOH}: \mathrm{H}_{2} \mathrm{O}(1: 1)$ at $60^{\circ} \mathrm{C}$ for overnight. After completion of the reductive cyclization, distilled out the $\mathrm{MeOH}$ and added water, product (5) was precipitated out and which was pure and reduced the purification. Product (5) was subject to $4 \mathrm{M} \mathrm{HCl}$ in dioxane at $0^{\circ} \mathrm{C}$ to $\mathrm{RT}$ for $2 \mathrm{~h}$ and isolated 2-(furan-2-yl)-1-(piperidin-4-yl)-1H-benzo[d] imidazole $\mathrm{HCl}$ salt (6).

2-(Furan-2-yl)-1-(piperidin-4-yl)-1H-benzo[d]imidazole $\mathrm{HCl}$ salt was derivatised with different acid, acyl chloride and sulphonyl chloride. For the acyl chlorides and sulphonyl chloride performed the reaction with TEA in THF at $0^{\circ} \mathrm{C}$ to RT for 3-4 hrs.

For acid-amine coupling, the standard procedure was employed by using HATU, DIPEA, and DMF at $0^{\circ} \mathrm{C}$ to RT for 2-3 hr. After completion of the reaction, the reaction mixture was quenched with ice cold water and the product was precipitated in a pure state without column purification

From the results of in vitro antimicrobial activity data indicate that the $\mathbf{7 c}, \mathbf{7 g}$ and $\mathbf{7 k}$ exhibited potent activity against E.coli, S.typhi, Micrococcus and B. megaterium and $\mathbf{7 a}, \mathbf{7 b}, \mathbf{7 d}$ and $\mathbf{7 h}$ showed moderate activity while others showed no or little activity. The compound $\mathbf{7 b}$ and $\mathbf{7 l}$ showed highest antimicrobial activity against all the bacterial species and fungal species due to the dichloro substituent and with sulfonamide and amide bond.

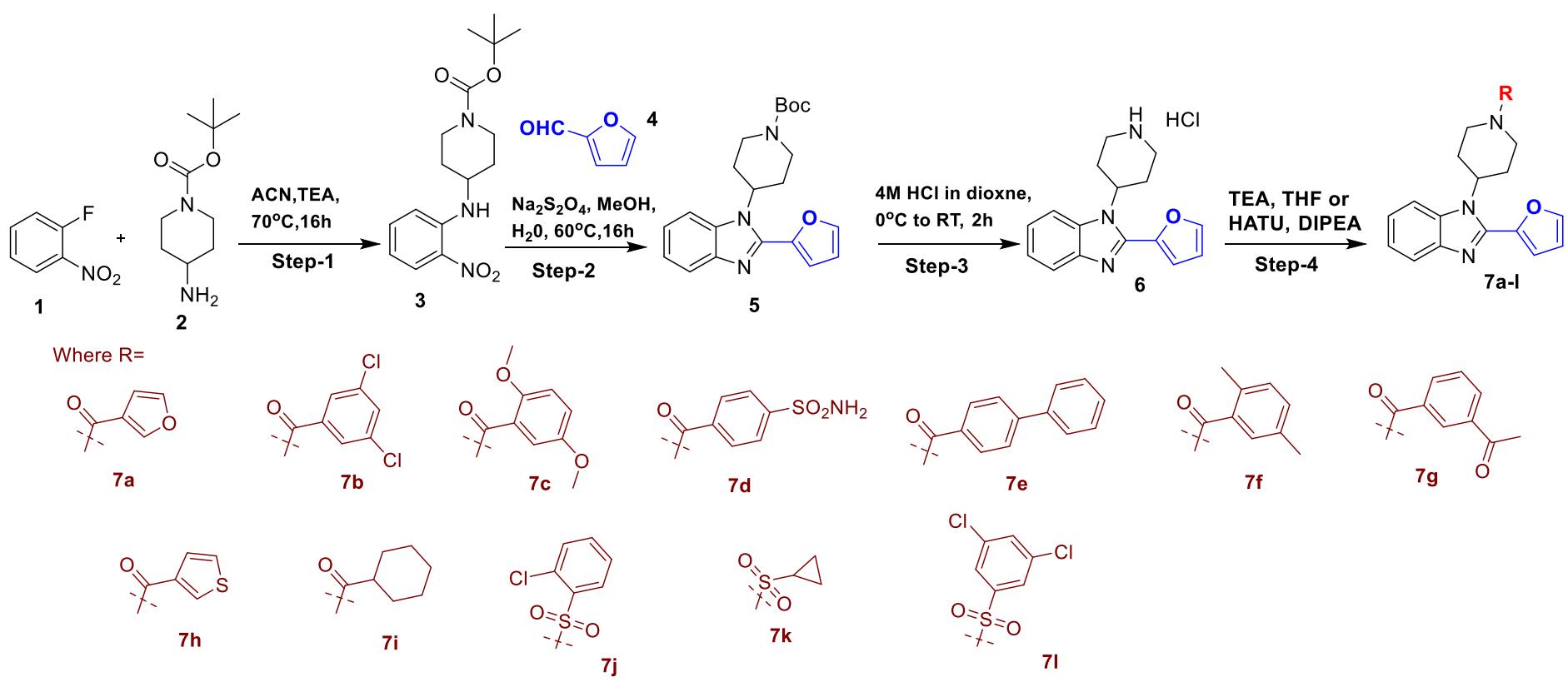

\section{Scheme 1}

Antimicrobial activities. Antibacterial and antifungal activity was tested by the standard agar cup method. All the synthesized compound (7a-7l) were tested for their in vitro antimicrobial activity against Gram +ve (Bacillus megaterium, Micrococcus spp.), Gram -ve (E.coli,, S. typi) and fungal spps. (Ganoderma spp., A.niger, A.flavus and Penicullium spp.) taking streptomycin, ciprofloxacin, and nystatin as standard drugs. A suspension of 24 to $48 \mathrm{~h}$ grown fresh bacterial and fungal culture was prepared in N-broth and Potato Dextrose broth 
respectively. All the bacterial and fungal suspensions were equally spread onto the sterile Muller Hinton and PDA respectively with the help of sterile swabs. Wells were made in the plates $(1 \mathrm{~cm})$ with the help of sterile cork borer. The standard antibiotics were dissolved in sterile distilled water up to $200 \mu \mathrm{g} / \mathrm{mL}$ of final concentration. The culture to be tested was dissolved in DMSO up to the final concentration of $1 \mathrm{mg} / \mathrm{mL}$ and $0.1 \mathrm{~mL}$ of it was loaded into the well. The plate was incubated at $4{ }^{\circ} \mathrm{C}$ for 20 minutes for proper diffusion of chemical and then the plates were incubated in upward position for $24 \mathrm{hrs}$ at $37{ }^{\circ} \mathrm{C}$ for bacterial culture and 48 hrs at $25{ }^{\circ} \mathrm{C}$ for fungal cultures. The control activity against DMSO was also performed. After an incubation zone of inhibition was observed and measured.

Table 1. Antimicrobial activity of 2-(furan-2-yl)-1-(piperidin-4-yl)-1H-benzo[d]imidazole derivatives

\begin{tabular}{|c|c|c|c|c|c|c|c|c|}
\hline \multirow{4}{*}{ Code } & \multicolumn{4}{|c|}{ Antibacterial activity } & \multicolumn{4}{|c|}{ Antifungal activity } \\
\hline & \multicolumn{4}{|c|}{$\begin{array}{c}\text { Antibacterial activity (zone in } \mathrm{cm} \text { ), } \\
\text { concentration: } 1 \mathrm{mg} / \mathrm{mL}\end{array}$} & \multirow{2}{*}{\multicolumn{4}{|c|}{$\begin{array}{l}\text { Antifungal activity (zone in } \mathrm{cm} \text { ), } \\
\text { concentration: } 1 \mathrm{mg} / \mathrm{mL}\end{array}$}} \\
\hline & \multicolumn{2}{|c|}{$\begin{array}{c}\text { Gram +ve } \\
\text { Bacteria }\end{array}$} & \multicolumn{2}{|c|}{$\begin{array}{l}\text { Gram -ve } \\
\text { Bacteria }\end{array}$} & & & & \\
\hline & $\begin{array}{c}\text { B. } \\
\text { megaterium }\end{array}$ & $\begin{array}{l}\text { Micrococcus } \\
\text { spp. }\end{array}$ & $\begin{array}{l}\text { S. } \\
\text { typi. }\end{array}$ & $\begin{array}{l}\text { E. } \\
\text { coli }\end{array}$ & $\begin{array}{l}\text { Penicillium } \\
\text { spp. }\end{array}$ & $\begin{array}{l}\text { Ganoderma } \\
\text { spp. }\end{array}$ & $\begin{array}{c}\text { A. } \\
\text { Niger }\end{array}$ & $\begin{array}{l}\text { A. } \\
\text { flavus }\end{array}$ \\
\hline $7 a$ & - & - & 1.8 & - & 1.2 & 0.9 & - & 0.5 \\
\hline $7 b$ & 2.5 & 3 & 2.1 & 2 & 2.8 & 3.2 & 3.0 & 3.5 \\
\hline 7c & 1.3 & 1.5 & 1.9 & - & 2.1 & 2.5 & 2.7 & 2.6 \\
\hline $7 d$ & 2 & 1.8 & 1.6 & 1.1 & 0.8 & 1.0 & 0.5 & - \\
\hline $7 e$ & 1.2 & 1 & - & 1.1 & 0.6 & - & 0.9 & 0.6 \\
\hline $7 f$ & 0.9 & 1.3 & 1.2 & 1.2 & - & 1.1 & 0.8 & 0.9 \\
\hline $7 g$ & 2.4 & 2.8 & 1.9 & 2.2 & 2.5 & 3.5 & 2.9 & 3.4 \\
\hline 7h & 1.2 & 1.9 & 1.7 & - & 1.5 & 1.9 & 2 & 1.8 \\
\hline $7 \mathbf{i}$ & - & 1.7 & 1.2 & 1.9 & 0.9 & 0.5 & 1.0 & - \\
\hline $7 \mathbf{j}$ & 1.5 & - & 1.2 & 1 & 2.1 & 3 & 2.5 & 2.9 \\
\hline $7 k$ & 2.6 & 2.9 & 1.8 & 2 & 1.0 & 0.7 & 0.5 & 0.8 \\
\hline 7I & 1.2 & - & 1.7 & - & 2.1 & 2.8 & 3.0 & 2.9 \\
\hline $\begin{array}{c}\text { Streptomycin } \\
(200 \mu \mathrm{g} / \mathrm{mL})\end{array}$ & 3 & 2 & 2 & 3.2 & - & - & - & - \\
\hline $\begin{array}{l}\text { Ciprofoxacin } \\
(200 \mu \mathrm{g} / \mathrm{mL})\end{array}$ & 3.8 & 4 & 4 & 3 & - & - & - & - \\
\hline $\begin{array}{l}\text { Nystatin } \\
200 \mu \mathrm{g} / \mathrm{ml}\end{array}$ & - & - & - & - & 3.2 & 4 & 3.5 & 3.8 \\
\hline
\end{tabular}

\section{Conclusions}

The aim of the present work was to synthesize, characterize and screen the antimicrobial activity of new 2(furan-2-yl)-1-(piperidin-4-yl)-1H-benzo[d]imidazole derivatives. The imidazole ring was cyclized via reductive 
cyclization with furfural and sodium dithionate. Compounds $\mathbf{7 b}$ and $\mathbf{7 l}$ show the highest antimicrobial activity against all species of bacteria and fungi.

\section{Experimental Section}

General. All purchased chemicals were used without further purification. Reactions were monitored by thin layer chromatography (TLC) on silica gel-G plates (G60 F254 (Merck)) of $0.5 \mathrm{~mm}$ thickness, visualizing with ultraviolet light (254 and $365 \mathrm{~nm}$ ), or with iodine vapor or aq. KMnO4. Melting points were determined Kofler hot plate apparatus. NMR spectra were recorded on a Bruker Avance $400 \mathrm{MHz}$ spectrometer $\left(400 \mathrm{MHz}\right.$ for ${ }^{1} \mathrm{H}$ $\mathrm{NMR}$ and $101 \mathrm{MHz}$ for ${ }^{13} \mathrm{C} \mathrm{NMR}$ ) respectively in deuterated solvents like $\mathrm{CDCl}_{3}$ (7.26) or DMSO- $d_{6}(2.5)$ and chemical shifts are referenced to the solvent residual signals with respect to tetramethylsilane; ${ }^{1} \mathrm{H} N M R$ chemical shifts are designated using the following abbreviations as well as their combinations: $s=\operatorname{singlet}, d=$ doublet, $\mathrm{t}=$ triplet, $\mathrm{q}=$ quartet, $\mathrm{m}=$ multiplet, $\mathrm{br}=$ broad signal, coupling constants in $\mathrm{Hz}$. Elemental analysis was carried out on Euro EA 3000 elemental analyser and the results are in agreement with the structures assigned. LCMS spectra were recorded on a Shimadzu LCMS-9030 spectrometer. Solvents were evaporated with a Büchi rotary evaporator. Purification was performed by column chromatography using silica gel (60-120 mesh size), borosil glass column having a length about $1000 \mathrm{~mm}$ and pet ether: ethyl acetate as a solvent system.

Tert-butyl 4-((2-nitrophenyl)amino)piperidine-1-carboxylate (3). 1-Fluoro-2-nitrobenzene 1 (3.0 g, 21.26 mmol) and tert-butyl 4-aminopiperidine-1-carboxylate $2(5.32 \mathrm{~g}, 26.58 \mathrm{mmol})$ was heated in the presence of TEA $(9.2 \mathrm{~mL}, 63.82 \mathrm{mmol})$ in ACN $(30 \mathrm{~mL})$ at $70{ }^{\circ} \mathrm{C}$ for $16 \mathrm{~h}$. Afterwards water $(100 \mathrm{~mL})$ was added and resulting mixture was extracted with ethyl acetate $(3 \times 50 \mathrm{~mL})$. The combine organic were dried over $\left(\mathrm{Na}_{2} \mathrm{SO}_{4}\right)$ and concentrated to dryness. The remaining crude mixture was chromatographed on a silica gel column using EtOAc/ $n$-hexane (3:7) to give 3 as a yellow liquid $(4.7 \mathrm{~g}, 70 \%) .{ }^{1} \mathrm{H}$ NMR (400 MHz, DMSO- $\left.d_{6}\right): \delta 8.08$ (dd, $J 1.2$, $8.8 \mathrm{~Hz}, 1 \mathrm{H}), 7.94(\mathrm{t}, J 18.8 \mathrm{~Hz}, 1 \mathrm{H}), 7.55(\mathrm{t}, J 14.8 \mathrm{~Hz}, 1 \mathrm{H}), 7.201$ (d, J $8.8 \mathrm{~Hz}, 1 \mathrm{H}), 3.83-3.92(\mathrm{~m}, 3 \mathrm{H}), 2.90-2.99$ $(\mathrm{m}, 4 \mathrm{H}), 1.92-1.98(\mathrm{~m}, 2 \mathrm{H}), 1.23(\mathrm{~s}, 9 \mathrm{H}) . \mathrm{MS}: \mathrm{m} / z$ [M-] 321.3.

tert-Butyl 4-(2-(furan-2-yl)-1H-benzo[d]imidazol-1-yl)piperidine-1-carboxylate $\quad$ (5). $\quad$ tert-Butyl $\quad 4-((2-$ nitrophenyl)amino)piperidine-1-carboxylate $3(3.0 \mathrm{~g}, 9.33 \mathrm{mmol})$ and furan-2-carbaldehyde $4(0.897 \mathrm{~g}, 9.33$ mmol) was added to methanol $(30 \mathrm{~mL})$, and $\mathrm{Na}_{2} \mathrm{~S}_{2} \mathrm{O}_{4}(4.87 \mathrm{~g}, 27.66 \mathrm{mmol})$ with water $(30 \mathrm{~mL})$ and reaction was heated at $70^{\circ} \mathrm{C}$ for $16 \mathrm{~h}$. Concentrated the reaction mixture and added water $(100 \mathrm{~mL})$ solid was precipitated filter it and dry it under vacuum to give $5(3.0 \mathrm{~g}, 87.4 \%)$ as a white solid. ${ }^{1} \mathrm{H}$ NMR (400 MHz, DMSO- $\left.d_{6}\right): \delta 1.52$ (s, 9H), $1.93(\mathrm{~d}, J 11.2 \mathrm{~Hz}, 2 \mathrm{H}), 2.25-2.35(\mathrm{~m}, 2 \mathrm{H}), 2.95(\mathrm{br}, 2 \mathrm{H}), 4.135(\mathrm{br}, 2 \mathrm{H}), 4.93-4.99(\mathrm{~m}, 1 \mathrm{H}), 6.77-6.79(\mathrm{~m}$, $1 \mathrm{H}), 7.16(\mathrm{~d}, J 3.6 \mathrm{~Hz}, 1 \mathrm{H}), 7.24-7.29(\mathrm{~m}, 2 \mathrm{H}), 7.69-7.70(\mathrm{~m}, 2 \mathrm{H}), 7.98-7.98(\mathrm{~d}, J 1.2 \mathrm{~Hz}, 1 \mathrm{H}) . \mathrm{MS}: m / z[M+1]$ 368.2 .

2-(Furan-2-yl)-1-(piperidin-4-yl)-1H-benzo[d]imidazole hydrochloride (6). tert-Butyl 4-(2-(furan-2-yl)-1Hbenzo[d]imidazol-1-yl)piperidine-1-carboxylate 5 (3.0 g, $2.61 \mathrm{mmol})$ was added in 1,4-dioxane $(30 \mathrm{~mL}) \mathrm{cool}$ at $0{ }^{\circ} \mathrm{C}$ was added $4 \mathrm{M} \mathrm{HCl}$ in dioxane drop wise and stirred at $\mathrm{rt}$ for $16 \mathrm{~h}$. Filtered the solid and dry it under vacuum to obtained $6(2.3 \mathrm{~g}, 92.73 \%)$ as an off white solid. ${ }^{1} \mathrm{H}$ NMR (400 MHz, DMSO-d $\left.d_{6}\right): \delta 2.22$ (d, br, J 8.0 $\mathrm{Hz}, 2 \mathrm{H}), 2.94$ (d, br, J $3.6 \mathrm{~Hz}, 2 \mathrm{H}), 3.21-3.30$ (m, 2H), 3.48 (d, br J 12.0 Hz, 2H), 5.27-5.29 (m, 1H), 6.98-6.99 (m, $1 \mathrm{H})$, 7.53-7.57 (m, 2H), 7.79-7.83 (m, 2H), $8.27(\mathrm{~s}, 1 \mathrm{H}), 8.53-8.55(\mathrm{~m}, 1 \mathrm{H}), 9.27-9.29(\mathrm{~m}, \mathrm{br}, 1 \mathrm{H}) \mathrm{HCl}$ salt and $9.91(\mathrm{~s}, \mathrm{br}, 1 \mathrm{H}) \mathrm{HCl}$ salt. MS: $m / z[\mathrm{M}+1] 268.1$. 
(4-(2-(Furan-2-yl)-1H-benzo[d]imidazol-1-yl)piperidin-1-yl)(furan-3-yl)methanone (7a). 2-(Furan-2-yl)-1(piperidin-4-yl)-1H-benzo[d]imidazole hydrochloride $6(150 \mathrm{mg}, 0.493 \mathrm{mmol})$ was added in THF (3 mL), trimethylamine $(0.21 \mathrm{~mL}, 1.48 \mathrm{mmol})$ cool to $0{ }^{\circ} \mathrm{C}$. furan-3-carbonyl chloride $(70.9 \mathrm{mg}, 0.543 \mathrm{mmol})$ was dilute with THF $(1 \mathrm{~mL})$ added drop wise at $0{ }^{\circ} \mathrm{C}$, stirred for $16 \mathrm{~h}$ at rt, extracted with ethyl acetate $(3 \times 20 \mathrm{~mL})$ and dried over anhydrous $\mathrm{Na}_{2} \mathrm{SO}_{4}$ and concentrated in vacuum. The crude product was purified by column chromatography on silica gel (eluent: $0-50 \% n$-Hexane/EtOAc) to afford the product $7 a$ as an off white solid $(0.1 \mathrm{~g}, 56 \%) ; \mathrm{mp} 183-186^{\circ} \mathrm{C} ;{ }^{1} \mathrm{H}$ NMR (400 MHz, DMSO- $\left.d_{6}\right): \delta$ 1.2-2.0 (m, 2H), 2.41-2.44 (m, 2H), 3.52-3.63 (m, $2 \mathrm{H}), 4.69-4.81(\mathrm{~m}, 2 \mathrm{H}), 5.04-5.10(\mathrm{~m}, 1 \mathrm{H}), 6.77(\mathrm{~s}, 1 \mathrm{H}), 6.79-6.80(\mathrm{~m}, 1 \mathrm{H}), 7.19(\mathrm{~d}, J 1.8 \mathrm{~Hz}, 1 \mathrm{H}), 7.25-7.30(\mathrm{~m}$, $2 \mathrm{H}), 7.68-7.70(\mathrm{~m}, 1 \mathrm{H}), 7.78(\mathrm{~s}, 1 \mathrm{H}), 7.82-7.84(\mathrm{~m}, 2 \mathrm{H}), 8.01(\mathrm{~s}, 1 \mathrm{H}), 8.144(\mathrm{~s}, 1 \mathrm{H}) ; \mathrm{LC}-\mathrm{MS}, \mathrm{RT}: 1.609$ min., $100 \%$, $\lambda \max : 308 \mathrm{~nm}$; MS: $\mathrm{m} / \mathrm{z}[\mathrm{M}+1]$ 362.3; Anal. Calc. for $\mathrm{C}_{21} \mathrm{H}_{19} \mathrm{~N}_{3} \mathrm{O}_{3}$ : C, 69.79; $\mathrm{H}, 5.30 ; \mathrm{N}, 11.63$; Found: C, 69.72; $\mathrm{H}, 5.25 ; \mathrm{N}, 11.60 \%$.

(3,5-Dichlorophenyl)(4-(2-(furan-2-yl)-1H-benzo[d]imidazol-1-yl)piperidin-1-yl)methanone (7b). 2-(Furan-2yl)-1-(piperidin-4-yl)-1H-benzo[d]imidazole hydrochloride 6 (150 mg, $0.493 \mathrm{mmol})$ was added in THF (3 mL), trimethylamine $(0.21 \mathrm{~mL}, 1.48 \mathrm{mmol}) \mathrm{cool}$ to $0{ }^{\circ} \mathrm{C}$. 3,5-dichlorobenzoyl chloride $(113.7 \mathrm{mg}, 0.543 \mathrm{mmol})$ was dilute with THF $(1 \mathrm{~mL})$ added drop wise at $0{ }^{\circ} \mathrm{C}$, stirred for $16 \mathrm{~h}$ at rt. Extracted with ethyl acetate $(3 \times 20 \mathrm{~mL})$ and dried over anhydrous $\mathrm{Na}_{2} \mathrm{SO}_{4}$ and concentrated in vacuum. The crude product was purified by column chromatography on silica gel (eluent: 0-60\% $n$-Hexane/EtOAc) to afford the product (3,5-dichlorophenyl)(4-(2(furan-2-yl)-1H-benzo[d]imidazol-1-yl)piperidin-1-yl)methanone as an off white solid (0.15 g, 69\%). mp 143$146{ }^{\circ} \mathrm{C} ;{ }^{1} \mathrm{H}$ NMR (400 MHz, DMSO- $\left.d_{6}\right): \delta$ 1.99-2.03 (m, 2H), 2.41-2.44 (m, 2H), 3.52-3.63 (m,2H), 4.69-4.81 (m, $2 \mathrm{H}), 5.04-5.10(\mathrm{~m}, 1 \mathrm{H}), 6.79-6.80(\mathrm{~m}, 1 \mathrm{H}), 7.18(\mathrm{~d}, J 3.2 \mathrm{~Hz}, 1 \mathrm{H}), 7.25-7.32(\mathrm{~m}, 2 \mathrm{H}), 7.63(\mathrm{~d}, J 2.0 \mathrm{~Hz}, 2 \mathrm{H}), 7.67-$ $7.69(\mathrm{~m}, 1 \mathrm{H}), 7.75(\mathrm{~d}, J 2.0 \mathrm{~Hz}, 1 \mathrm{H}), 7.99-8.03(\mathrm{~m}, 2 \mathrm{H})$; LC-MS, RT: $2.012 \mathrm{~min}, 98.96 \%, \lambda \max : 254 \mathrm{~nm} ; \mathrm{MS}: \mathrm{m} / \mathrm{z}$ [M+1] 442.2; Anal. Calc. for $\mathrm{C}_{23} \mathrm{H}_{19} \mathrm{Cl}_{2} \mathrm{~N}_{3} \mathrm{O}_{2}: \mathrm{C}, 62.74 ; \mathrm{H}, 4.35 ; \mathrm{N}, 9.54$; Found: $\mathrm{C}, 62.70 ; \mathrm{H}, 4.31 ; \mathrm{N}, 9.49 \%$.

(2,5-Dimethoxyphenyl)(4-(2-(furan-2-yl)-1H-benzo[d]imidazol-1-yl)piperidin-1-yl)methanone (7c). 2,5dimethoxybenzoic acid $\left(98.95 \mathrm{mg}, 0.543 \mathrm{mmol}\right.$ ) was added in DMF $(3 \mathrm{~mL})$ cool to $0^{\circ} \mathrm{C}$, HATU $(281 \mathrm{mg}, 0.74$ mmol) was added and stirred for $30 \mathrm{~min}$ at $0^{\circ} \mathrm{C}$. 2-(furan-2-yl)-1-(piperidin-4-yl)-1H-benzo[d]imidazole hydrochloride $6\left(150 \mathrm{mg}, 0.493 \mathrm{mmol}\right.$ ) was added portion wise and stirred for $30 \mathrm{~min}$ at $0^{\circ} \mathrm{C}$. DIPEA (0.27 mL, $0.156 \mathrm{mmol})$ was added dropwise at $0{ }^{\circ} \mathrm{C}$ and stirred for $16 \mathrm{~h}$. Extracted with ethyl acetate $(3 \times 15 \mathrm{~mL})$ and dried over anhydrous $\mathrm{Na}_{2} \mathrm{SO}_{4}$ and concentrated in vacuum. The crude product was purified by column chromatography on silica gel (eluent: 0-60\% $n$-Hexane/EtOAc) to afford the product (2,5-dimethoxyphenyl)(4(2-(furan-2-yl)-1H-benzo[d]imidazol-1-yl)piperidin-1-yl)methanone as yellow solid (150 mg, 70.4\%); mp 110$114{ }^{\circ} \mathrm{C} ;{ }^{1} \mathrm{H}$ NMR $\left(400 \mathrm{MHz}, \mathrm{DMSO}-d_{6}\right): \delta 1.83-1.90(\mathrm{~m}, 2 \mathrm{H}), 2.06-2.10(\mathrm{~m}, 2 \mathrm{H}), 2.90-2.98(\mathrm{~m}, 1 \mathrm{H}), 3.18-3.25(\mathrm{~m}$, $1 \mathrm{H}), 3.44-3.50(\mathrm{~m}, 1 \mathrm{H}), 3.74-377(\mathrm{~m}, 4 \mathrm{H}), 3.88(\mathrm{~s}, 2 \mathrm{H}),\left(4-\mathrm{OCH}_{3}\right.$ split in $4 \mathrm{H}$ and $\left.2 \mathrm{H}\right), 4.73-4.76(\mathrm{~m}, 1 \mathrm{H}), 5.10(\mathrm{~s}$, br, $1 \mathrm{H}), 6.79(\mathrm{~s}, 1 \mathrm{H}), 6.96(\mathrm{~d}, J 1.2 \mathrm{~Hz}, 1 \mathrm{H}), 6.98-7.05(\mathrm{~m}, 1 \mathrm{H}), 7.16-7.19(\mathrm{~m}, 1 \mathrm{H}), 7.27-7.31(\mathrm{~m}, 1 \mathrm{H}), 7.36-7.40$ $(\mathrm{m}, 1 \mathrm{H}), 7.66-7.72(\mathrm{~m}, 1 \mathrm{H}), 7.89-7.91(\mathrm{~m}, 1 \mathrm{H}), 8.01(\mathrm{~s}, 1 \mathrm{H}) ;{ }^{13} \mathrm{C}-\mathrm{NMR}\left(101 \mathrm{MHz}, \mathrm{DMSO}-d_{6}\right): \delta 166.49,153.68$, $149.45,149.33,145.55,145.49,144.80,144.73,144.02,143.84,143.60,134.12,133.88,126.95,126.90$, $123.18,122.74,120.44,120.06,115.30,115.22,113.90,113.80,113.51,113.07,112.81,112.54,56.57,56.45$, 56.00, 55.00, 54.52, 46.39, 46.00, 30.58, 30.46, 30.23, 30.02; LC-MS, RT: 1.602 min, 100\%, גmax: 302 nm; MS: $m / z$ [M+1] 432.4; Anal. Calc. for $\mathrm{C}_{25} \mathrm{H}_{25} \mathrm{~N}_{3} \mathrm{O}_{4}$ : C, 69.59; $\mathrm{H}, 5.84 ; \mathrm{N}, 9.74 ;$ Found: $\mathrm{C}, 69.53 ; \mathrm{H}, 5.79 ; \mathrm{N}, 9.69 \%$.

4-(4-(2-(Furan-2-yl)-1H-benzo[d]imidazol-1-yl)piperidine-1-carbonyl)benzenesulfonamide (7d). 4sulfamoylbenzoic acid (109.2 $\mathrm{mg}, 0.543 \mathrm{mmol}$ ) was added in DMF (3 mL) cool to $0{ }^{\circ} \mathrm{C}$, HATU $(281 \mathrm{mg}, 0.74$ mmol) was added and stirred for $30 \mathrm{~min}$ at $0{ }^{\circ} \mathrm{C}$. 2-(furan-2-yl)-1-(piperidin-4-yl)-1H-benzo[ $\left.d\right]$ imidazole hydrochloride 6 (150 mg, $0.493 \mathrm{mmol})$ was added portion wise and stirred for $30 \mathrm{~min}$ at $0{ }^{\circ} \mathrm{C}$. DIPEA (0.27 mL, $0.156 \mathrm{mmol})$ was added dropwise at $0{ }^{\circ} \mathrm{C}$ and stirred for $16 \mathrm{~h}$. Extracted with ethyl acetate $(3 \times 15 \mathrm{~mL})$ and dried over anhydrous $\mathrm{Na}_{2} \mathrm{SO}_{4}$ and concentrated in vacuum. The crude product was purified by column 
chromatography on silica gel (eluent: $0-70 \% n$-Hexane/EtOAc) to afford the product 4-(4-(2-(furan-2-yl)- $1 H$ benzo[d]imidazol-1-yl)piperidine-1-carbonyl)benzenesulfonamide as yellow solid (140 $\mathrm{mg}, 65.71 \%$ ); $\mathrm{mp} 248$ $251{ }^{\circ} \mathrm{C} ;{ }^{1} \mathrm{H}$ NMR $\left(400 \mathrm{MHz}\right.$, DMSO- $\left.d_{6}\right): \delta$ 1.83-1.90 (m, 2H), 1.92-2.05 (m, 3H), $2.51(\mathrm{~m}, 1 \mathrm{H}), 2.90-3.01(\mathrm{~m}, 2 \mathrm{H})$, $3.64(\mathrm{~m}, 1 \mathrm{H}), 4.73(\mathrm{~m}, 1 \mathrm{H}), 5.06(\mathrm{~s}, \mathrm{br}, 1 \mathrm{H}), 6.79(\mathrm{~s}, \mathrm{br}, 1 \mathrm{H}), 7.17-7.29(\mathrm{~m}, 3 \mathrm{H}), 7.48(\mathrm{~m}, 2 \mathrm{H}), 7.73(\mathrm{~m}, 2 \mathrm{H}), 7.93$ (m, 4H); LC-MS, RT: $1.463 \mathrm{~min}, 100 \%, \lambda \max : 254 \mathrm{~nm}$; MS: $\mathrm{m} / z$ [M+1] 451.3; Anal. Calc. for $\mathrm{C}_{23} \mathrm{H}_{22} \mathrm{~N}_{4} \mathrm{O}_{4} \mathrm{~S}: \mathrm{C}_{\text {, }}$ 61.32; $\mathrm{H}, 4.92 ; \mathrm{N}, 12.44$; Found: $\mathrm{C}, 61.26 ; \mathrm{H}, 4.85 ; \mathrm{N}, 12.38 \%$.

[1,1'-Biphenyl]-4-yl(4-(2-(furan-2-yl)-1H-benzo[d]imidazol-1-yl)piperidin-1-yl)methanone (7e). [1,1'biphenyl]-4-carboxylicacid (107.6 mg, $0.543 \mathrm{mmol}$ ) was added in DMF $(3 \mathrm{~mL})$ cool to $0{ }^{\circ} \mathrm{C}$, HATU $(281 \mathrm{mg}, 0.74$ $\mathrm{mmol}$ ) was added and stirred for $30 \mathrm{~min}$ at $0{ }^{\circ} \mathrm{C}$. 2-(furan-2-yl)-1-(piperidin-4-yl)-1H-benzo[d]imidazole hydrochloride 6 (150 mg, $0.493 \mathrm{mmol}$ ) was added portion wise and stirred for $30 \mathrm{~min}$ at $0{ }^{\circ} \mathrm{C}$. DIPEA (0.27 mL, $0.156 \mathrm{mmol})$ was added dropwise at $0{ }^{\circ} \mathrm{C}$ and stirred for $16 \mathrm{~h}$. Extracted with ethyl acetate $(3 \times 15 \mathrm{~mL})$ and dried over anhydrous $\mathrm{Na}_{2} \mathrm{SO}_{4}$ and concentrated in vacuum. The crude product was purified by column chromatography on silica gel (eluent: $0-50 \% n$-Hexane/EtOAc) to afford the product [1,1'-biphenyl]-4-yl(4-(2(furan-2-yl)-1H-benzo[d]imidazol-1-yl)piperidin-1-yl)methanone as yellow solid (170 mg, 79.79\%); mp 128-131 ${ }^{\circ} \mathrm{C} ;{ }^{1} \mathrm{H}$ NMR (400 MHz, DMSO- $\left.d_{6}\right): \delta 1.24-1.34(\mathrm{~m}, 3 \mathrm{H}), 1.97(\mathrm{~s}, \mathrm{br}, 2 \mathrm{H}), 3.01(\mathrm{~m}, 1 \mathrm{H}), 3.85(\mathrm{~m}, 1 \mathrm{H}), 4.74(\mathrm{~m}, 1 \mathrm{H})$, $5.08(\mathrm{~s}, \mathrm{br}, 1 \mathrm{H}), 6.80(\mathrm{~s}, \mathrm{br}, 1 \mathrm{H}), 7.18(\mathrm{~s}, \mathrm{br}, 1 \mathrm{H}), 7.29(\mathrm{~s}, \mathrm{br}, 2 \mathrm{H}), 7.41-7.50(\mathrm{~m}, 3 \mathrm{H}), 7.64(\mathrm{~s}, 2 \mathrm{H}), 7.72-7.77(\mathrm{~m}$, 5H),7.92 (s, 1H), $8.03(\mathrm{~s}, 1 \mathrm{H}) ;{ }^{13} \mathrm{C}-\mathrm{NMR}\left(101 \mathrm{MHz}\right.$, DMSO- $\left.d_{6}\right): \delta 169.51,145.52,144.88,144.06,143.66,141.69$, $139.86,135.54,134.16,129.53,128.36,128.12,127.29,127.19,123.28,122.75,120.11,113.94,113.49$, 112.60, 55.08, 54.05, 47.16, 41.65, 30.63, 29.89, 29.51, 18.55, 17.19; LC-MS, RT: 1.788 min, 99\%, $\lambda \operatorname{max:~} 254$ nm; MS: $m / z[M+1]$ 448.4; Anal. Calc. for $\mathrm{C}_{29} \mathrm{H}_{25} \mathrm{~N}_{3} \mathrm{O}_{2}$ : C, 77.83; $\mathrm{H}, 5.63 ; \mathrm{N}, 9.39$; Found: $\mathrm{C}, 77.76 ; \mathrm{H}, 5.59 ; \mathrm{N}$, $9.33 \%$.

(2,5-Dimethylphenyl) (4-(2-(furan-2-yl)-1H-benzo[d]imidazol-1-yl)piperidin-1-yl)methanone (7f). 2,5dimethylbenzoic acid $(82 \mathrm{mg}, 0.543 \mathrm{mmol})$ was added in DMF $(3 \mathrm{~mL})$ cool to $0{ }^{\circ} \mathrm{C}$, HATU $(281 \mathrm{mg}, 0.74 \mathrm{mmol})$ was added and stirred for $30 \mathrm{~min}$ at $0{ }^{\circ} \mathrm{C}$. 2-(furan-2-yl)-1-(piperidin-4-yl)-1H-benzo[d]imidazole hydrochloride $6\left(150 \mathrm{mg}, 0.493 \mathrm{mmol}\right.$ ) was added portion wise and stirred for $30 \mathrm{~min}$ at $0{ }^{\circ} \mathrm{C}$. DIPEA $(0.27 \mathrm{~mL}, 0.156 \mathrm{mmol})$ was added dropwise at $0{ }^{\circ} \mathrm{C}$ and stirred for $16 \mathrm{~h}$. Extracted with ethyl acetate $(3 \times 15 \mathrm{~mL})$ and dried over anhydrous $\mathrm{Na}_{2} \mathrm{SO}_{4}$ and concentrated in vacuum. The crude product was purified by column chromatography on silica gel (eluent: $0-70 \% n$-Hexane/EtOAc) to afford the product (2,5-dimethylphenyl)(4-(2-(furan-2-yl)-1Hbenzo[d]imidazol-1-yl)piperidin-1-yl)methanone as yellow solid (155 mg, 72.75\%); mp 150-154 ${ }^{\circ} \mathrm{C} ;{ }^{1} \mathrm{H}$ NMR (400 MHz, DMSO- $\left.d_{6}\right): \delta 1.25(\mathrm{~m}, 6 \mathrm{H}), 1.86(\mathrm{~s}, \mathrm{br}, 1 \mathrm{H}), 2.09(\mathrm{~s}, \mathrm{br}, 1 \mathrm{H}), 2.32(\mathrm{~m}, 2 \mathrm{H}), 2.96-3.02(\mathrm{~m}, 1 \mathrm{H}), 3.23-3.29$ $(\mathrm{m}, 1 \mathrm{H}), 3.63(\mathrm{~s}, \mathrm{br}, 1 \mathrm{H}), 4.77(\mathrm{~s}, \mathrm{br}, 1 \mathrm{H}), 5.049(\mathrm{~s}, 1 \mathrm{H}), 6.68(\mathrm{~d}, J 7.2 \mathrm{~Hz}, 1 \mathrm{H}), 6.99(\mathrm{~s}, 1 \mathrm{H}), 7.14-7.29(\mathrm{~m}, 5 \mathrm{H})$, 7.68-7.70 (m, 1H), 7.89-8.03 (m, 2H); LC-MS, RT: $1.675 \mathrm{~min}, 95.43 \%, \lambda \max : 202 \mathrm{~nm}, \mathrm{MS}: \mathrm{m} / z$ [M+1] 400.4; Anal. Calc. for $\mathrm{C}_{25} \mathrm{H}_{25} \mathrm{~N}_{3} \mathrm{O}_{2}$ : C, 75.16; $\mathrm{H}, 6.31 ; \mathrm{N}, 10.52$; Found: $\mathrm{C}, 75.10 ; \mathrm{H}, 6.25 ; \mathrm{N}, 10.47 \%$.

(4-(2-(Furan-2-yl)-1H-benzo[d]imidazol-1-yl)piperidin-1-yl)(thiophen-3-yl)methanone (7g). 3-acetylbenzoic acid ( $89 \mathrm{mg}, 0.543 \mathrm{mmol}$ ) was added in DMF $(3 \mathrm{~mL})$ cool to $0^{\circ} \mathrm{C}$, HATU $(281 \mathrm{mg}, 0.74 \mathrm{mmol})$ was added and stirred for $30 \mathrm{~min}$ at $0{ }^{\circ} \mathrm{C}$. 2-(furan-2-yl)-1-(piperidin-4-yl)-1H-benzo[d]imidazole hydrochloride 6 (150 mg, $0.493 \mathrm{mmol}$ ) was added portion wise and stirred for $30 \mathrm{~min}$ at $0{ }^{\circ} \mathrm{C}$. DIPEA $(0.27 \mathrm{~mL}, 0.156 \mathrm{mmol})$ was added dropwise at $0{ }^{\circ} \mathrm{C}$ and stirred for $16 \mathrm{~h}$. Extracted with ethyl acetate $(3 \times 15 \mathrm{~mL})$ and dried over anhydrous $\mathrm{Na}_{2} \mathrm{SO}_{4}$ and concentrated in vacuum. The crude product was purified by column chromatography on silica gel (eluent: $0-60 \% n$-Hexane/EtoAc) to afford the product (4-(2-(furan-2-yl)-1H-benzo[d]imidazol-1-yl)piperidin-1$\mathrm{yl}$ )(thiophen-3-yl)methanone as yellow solid (145 mg, 68\%); mp 155-158 ${ }^{\circ} \mathrm{C} ;{ }^{1} \mathrm{H}$ NMR (400 MHz, DMSO-d $\left.{ }_{6}\right): \delta$ 1.91-2.06 (m, 4H), $2.64(\mathrm{~s}, 3 \mathrm{H}), 3.02(\mathrm{~m}, 2 \mathrm{H}), 3.71(\mathrm{~m}, 1 \mathrm{H}), 4.75(\mathrm{~m}, 1 \mathrm{H}), 5.07(\mathrm{~S}, 1 \mathrm{H}), 6.80(\mathrm{~S}, 1 \mathrm{H}), 7.19(\mathrm{~d}, J 2.8$ $\mathrm{Hz}, 1 \mathrm{H}), 7.25-7.32(\mathrm{~m}, 2 \mathrm{H}), 7.64-7.70(\mathrm{~m}, 2 \mathrm{H}), 7.81(\mathrm{~d}, J 7.2 \mathrm{~Hz}, 1 \mathrm{H}), 7.95(\mathrm{~d}, J 7.2 \mathrm{~Hz}, 1 \mathrm{H}), 8.03-8.07(\mathrm{~m}, 3 \mathrm{H})$; LC- 
MS, RT: 1.727 min, 90.8\%, $\lambda \max : 254 \mathrm{~nm}$; MS: $m / z$ [M+1] 414.2; Anal. Calc. for $\mathrm{C}_{25} \mathrm{H}_{23} \mathrm{~N}_{3} \mathrm{O}_{3}: \mathrm{C}, 72.62 ; \mathrm{H}, 5.61$; N, 10.16; Found: C, 72.57; H, 5.56; N, 10.10\%.

(4-(2-(Furan-2-yl)-1H-benzo[d]imidazol-1-yl)piperidin-1-yl)(thiophen-3-yl)methanone (7h). Thiophene-3carboxylic acid (69 mg, $0.543 \mathrm{mmol}$ ) was added in DMF $(3 \mathrm{~mL})$ cool to $0{ }^{\circ} \mathrm{C}$, HATU $(281 \mathrm{mg}, 0.74 \mathrm{mmol})$ was added and stirred for $30 \mathrm{~min}$ at $0{ }^{\circ} \mathrm{C}$. 2-(furan-2-yl)-1-(piperidin-4-yl)-1H-benzo[d]imidazole hydrochloride 6 (150 mg, $0.493 \mathrm{mmol}$ ) was added portion wise and stirred for $30 \mathrm{~min}$ at $0{ }^{\circ} \mathrm{C}$. DIPEA $(0.27 \mathrm{~mL}, 0.156 \mathrm{mmol})$ was added dropwise at $0{ }^{\circ} \mathrm{C}$ and stirred for $16 \mathrm{~h}$. Extracted with ethyl acetate $(3 \times 15 \mathrm{~mL})$ and dried over anhydrous $\mathrm{Na}_{2} \mathrm{SO}_{4}$ and concentrated in vacuum. The crude product was purified by column chromatography on silica gel (eluent: $0-75 \% n$-Hexane/EtOAc) to afford the product (4-(2-(furan-2-yl)-1H-benzo[d]imidazol-1yl)piperidin-1-yl)(thiophen-3-yl)methanone as yellow solid (130 mg, 69.7\%); mp 172-176 ${ }^{\circ} \mathrm{C} ;{ }^{1} \mathrm{H}$ NMR (400 MHz, DMSO- $\left.d_{6}\right): \delta 2.00(\mathrm{~m}, 2 \mathrm{H}), 2.40-2.43(\mathrm{~m}, 2 \mathrm{H}), 3.04(\mathrm{~m}, 2 \mathrm{H}), 4.04(\mathrm{~m}, 1 \mathrm{H}), 4.67(\mathrm{~m}, 1 \mathrm{H}), 5.066(\mathrm{t}, J 24.0 \mathrm{~Hz}$, $1 \mathrm{H}), 6.79(\mathrm{~s}, 1 \mathrm{H}), 7.18(\mathrm{~d}, J 2.8 \mathrm{~Hz}, 1 \mathrm{H}), 7.24-7.33(\mathrm{~m}, 3 \mathrm{H}), 7.65-7.67(\mathrm{~m}, 2 \mathrm{H}), 7.86-7.91(\mathrm{~m}, 2 \mathrm{H}), 8.01(\mathrm{~s}, 1 \mathrm{H})$; LC-

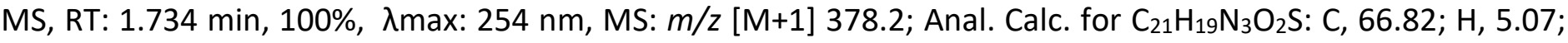
N, 11.13; Found: C, 66.77; H, 5.01; N, 11.07\%.

Cyclohexyl (4-(2-(furan-2-yl)-1H-benzo[d]imidazol-1-yl)piperidin-1-yl)methanone (7i). Cyclohexanecarboxylic acid $(69 \mathrm{mg}, 0.543 \mathrm{mmol})$ was added in DMF $(3 \mathrm{~mL})$ cool to $0{ }^{\circ} \mathrm{C}$, HATU $(281 \mathrm{mg}, 0.74 \mathrm{mmol})$ was added and stirred for $30 \mathrm{~min}$ at $0{ }^{\circ} \mathrm{C}$. 2-(furan-2-yl)-1-(piperidin-4-yl)-1H-benzo[d]imidazole hydrochloride 6 (150 mg, $0.493 \mathrm{mmol}$ ) was added portion wise and stirred for $30 \mathrm{~min}$ at $0{ }^{\circ} \mathrm{C}$. DIPEA $(0.27 \mathrm{~mL}, 0.156 \mathrm{mmol})$ was added dropwise at $0{ }^{\circ} \mathrm{C}$ and stirred for $16 \mathrm{~h}$. Extracted with ethyl acetate $(3 \times 15 \mathrm{~mL})$ and dried over anhydrous $\mathrm{Na}_{2} \mathrm{SO}_{4}$ and concentrated in vacuum. The crude product was purified by column chromatography on silica gel (eluent: $0-70 \% n$-Hexane/EtOAc) to afford the product cyclohexyl(4-(2-(furan-2-yl)-1H-benzo[d]imidazol-1yl)piperidin-1-yl)methanone as yellow solid (130 mg, 69.7\%); mp 208-212 ${ }^{\circ} \mathrm{C} ;{ }^{1} \mathrm{H}$ NMR (400 MHz, DMSO- $\left.d_{6}\right): \delta$ 1.17-1.47 (m, 6H), 1.67-1.76 (m, 4H), 1.97-2.09 (m, 2H), 2.20-2.22 (m, 2H), $2.68(\mathrm{~m}, \mathrm{br}, 2 \mathrm{H}), 3.16-3.22(\mathrm{~m}, 1 \mathrm{H})$, 4.13-4.16 (m, 1H), $4.63(\mathrm{~d}, J 12.0 \mathrm{~Hz}, 1 \mathrm{H}), 5.02(\mathrm{t}, J 24.0 \mathrm{~Hz}, 1 \mathrm{H}), 6.78(\mathrm{t}, J 3.2 \mathrm{~Hz}, 1 \mathrm{H}), 7.16(\mathrm{~d}, J 3.2 \mathrm{~Hz}, 1 \mathrm{H})$, 7.22-7.28 (m, 2H), 7.67-7.69 (m, 2H), $7.98(\mathrm{~s}, 1 \mathrm{H}) ;{ }^{13} \mathrm{C}-\mathrm{NMR}\left(101 \mathrm{MHz}, \mathrm{DMSO}-d_{6}\right): \delta$ 173.96, 145.43, 144.89, 144.03, 143.64, 134.16, 123.23, 122.70, 120.17, 113.90, 113.07, 112.56, 55.11, 44.73, 31.30, 30.40, 29.72, 26.12, 25.69; LC-MS, RT: 1.624 min, 100\%, $\lambda \max : 247 \mathrm{~nm}, \mathrm{MS}: \mathrm{m} / z$ [M+1] 378.4; Anal. Calc. for $\mathrm{C}_{23} \mathrm{H}_{27} \mathrm{~N}_{3} \mathrm{O}_{2}: \mathrm{C}_{\text {, }}$ 73.18; H, 7.21; N, 11.13; Found: C, 73.12; H, 7.17; N, 11.08\%.

1-(1-((2-Chlorophenyl)sulfonyl)piperidin-4-yl)-2-(furan-2-yl)-1H-benzo[d]imidazole (7j). 2-(Furan-2-yl)-1(piperidin-4-yl)-1H-benzo[d]imidazole hydrochloride $6(150 \mathrm{mg}, 0.493 \mathrm{mmol}$ ) was added in THF (3 $\mathrm{mL}$ ), trimethylamine $(0.21 \mathrm{~mL}, 1.48 \mathrm{mmol})$ cool to $0{ }^{\circ} \mathrm{C}$. 2-chlorobenzenesulfonyl chloride $(114.6 \mathrm{mg}, 0.543 \mathrm{mmol})$ was dilute with THF $(1 \mathrm{~mL})$ added drop wise at $0{ }^{\circ} \mathrm{C}$, stirred for $16 \mathrm{~h}$ at rt. Extracted with ethyl acetate $(3 \times 20$ $\mathrm{mL}$ ) and dried over anhydrous $\mathrm{Na}_{2} \mathrm{SO}_{4}$ and concentrated in vacuum. The crude product was purified by column chromatography on silica gel (eluent: $0-50 \%$ DCM/EtOAc) to afford the product 1-(1-((2chlorophenyl)sulfonyl)piperidin-4-yl)-2-(furan-2-yl)-1H-benzo[d]imidazole as an off white solid (0.14 g, 64.1\%); $\mathrm{mp} 128-132{ }^{\circ} \mathrm{C} ;{ }^{1} \mathrm{H}$ NMR (400 MHz, DMSO-d $): \delta 1.98-2.05(\mathrm{~m}, 2 \mathrm{H}), 2.34-2.40(\mathrm{~m}, 2 \mathrm{H}), 3.11(\mathrm{t}, J 24.0 \mathrm{~Hz}, 2 \mathrm{H})$, $3.97(\mathrm{~d}, J 12.0 \mathrm{~Hz}, 2 \mathrm{H}), 4.95(\mathrm{t}, J 12.4 \mathrm{~Hz}, 1 \mathrm{H}), 6.75-6.76(\mathrm{~m}, 1 \mathrm{H}), 7.15(\mathrm{~d}, J 3.2 \mathrm{~Hz}, 1 \mathrm{H}), 7.24-7.27(\mathrm{~m}, 2 \mathrm{H}), 7.40-$ $7.42(\mathrm{~m}, 1 \mathrm{H}), 7.62-7.69(\mathrm{~m}, 2 \mathrm{H}), 7.75-7.83(\mathrm{~m}, 1 \mathrm{H}), 7.96(\mathrm{~d}, J 1.2 \mathrm{~Hz}, 1 \mathrm{H}), 8.10(\mathrm{dd}, J 1.6,8.0 \mathrm{~Hz}, 1 \mathrm{H})$; LC-MS, RT: $1.969 \mathrm{~min}, 98.27 \%, \lambda \max : 254 \mathrm{~nm}, \mathrm{MS}: \mathrm{m} / \mathrm{z}[\mathrm{M}+1]$ 442.2; Anal. Calc. for $\mathrm{C}_{22} \mathrm{H}_{20} \mathrm{ClN}_{3} \mathrm{O} 3 \mathrm{~S}: \mathrm{C}, 59.79 ; \mathrm{H}, 4.56 ; \mathrm{N}$, 9.51; Found: C, 59.75; $\mathrm{H}, 4.50 ; \mathrm{N}, 9.45 \%$.

\section{1-(1-(Cyclopropylsulfonyl)piperidin-4-yl)-2-(furan-2-yl)-1H-benzo[d]imidazole (7k). 2-(Furan-2-yl)-1-} (piperidin-4-yl)-1H-benzo[d]imidazole hydrochloride $6(150 \mathrm{mg}, 0.493 \mathrm{mmol}$ ) was added in THF (3 $\mathrm{mL})$, trimethylamine $(0.21 \mathrm{~mL}, 1.48 \mathrm{mmol})$ cool to $0^{\circ} \mathrm{C}$. Cyclopropanesulfonyl chloride $(76.35 \mathrm{mg}, 0.543 \mathrm{mmol})$ was dilute with THF $(1 \mathrm{~mL})$ added drop wise at $0^{\circ} \mathrm{C}$, stirred for $16 \mathrm{~h}$ at rt. Extracted with ethyl acetate $(3 \times 20 \mathrm{~mL})$ 
and dried over anhydrous $\mathrm{Na}_{2} \mathrm{SO}_{4}$ and concentrated in vacuum. The crude product was purified by column chromatography on silica gel (eluent: $0-50 \%$ DCM/EtOAc) to afford the product 1-(1(cyclopropylsulfonyl)piperidin-4-yl)-2-(furan-2-yl)-1H-benzo[d]imidazole as an off white solid $(0.110 \mathrm{~g}$, 59.97\%); mp 117-120 ${ }^{\circ} \mathrm{C} ;{ }^{1} \mathrm{H}$ NMR (400 MHz, DMSO- $\left.d_{6}\right): \delta$ 0.98-1.00 (m, $\left.2 \mathrm{H}\right), 1.04-1.08(\mathrm{~m}, 2 \mathrm{H}), 2.04-2.06(\mathrm{~m}$, $2 \mathrm{H}), 2.34-2.40(\mathrm{~m}, 2 \mathrm{H}), 2.69-2.75(\mathrm{~m}, 1 \mathrm{H}), 3.11(\mathrm{t}, J 23.2 \mathrm{~Hz}, 2 \mathrm{H}), 3.83(\mathrm{~d}, J 12.0 \mathrm{~Hz}, 2 \mathrm{H}), 4.91-4.98(\mathrm{~m}, 1 \mathrm{H}), 6.78-$ $6.79(\mathrm{~m}, 1 \mathrm{H}), 7.18(\mathrm{~d}, J 3.6 \mathrm{~Hz}, 1 \mathrm{H}), 7.24-7.31(\mathrm{~m}, 2 \mathrm{H}), 7.68(\mathrm{~d}, J 2.0 \mathrm{~Hz}, 1 \mathrm{H}), 7.75-7.77(\mathrm{~m}, 2 \mathrm{H}), 8.01(\mathrm{~d}, J 1.2 \mathrm{~Hz}$, $1 \mathrm{H}) ;{ }^{13} \mathrm{C}-\mathrm{NMR}\left(101 \mathrm{MHz}\right.$, DMSO- $\left.d_{6}\right): \delta 145.56,144.78,144.12,143.68,134.05,123.33,122.74,120.19,113.93$, 113.20, 112.55, 54.46, 46.08, 30.15, 26.15, 4.61; LC-MS, RT: 1.793 min, 98.37\%, $\lambda$ max: 254 nm, MS: $m / z$ [M+1] 372.2; Anal. Calc. for $\mathrm{C}_{19} \mathrm{H}_{21} \mathrm{~N}_{3} \mathrm{O}_{3} \mathrm{~S}: \mathrm{C}, 61.44 ; \mathrm{H}, 5.70 ; \mathrm{N}, 11.31$; Found: $\mathrm{C}, 61.40 ; \mathrm{H}, 5.65 ; \mathrm{N}, 11.25 \%$.

1-(1-((3,5-Dichlorophenyl)sulfonyl)piperidin-4-yl)-2-(furan-2-yl)-1H-benzo[d]imidazole (7l). 2-(Furan-2-yl)-1(piperidin-4-yl)-1H-benzo[d]imidazole hydrochloride $6(150 \mathrm{mg}, 0.493 \mathrm{mmol})$ was added in THF $(3 \mathrm{~mL})$, trimethylamine $(0.21 \mathrm{~mL}, 1.48 \mathrm{mmol})$ cool to $0{ }^{\circ} \mathrm{C}$. 3,5-dichlorobenzenesulfonyl chloride $(133.3 \mathrm{mg}, 0.543$ $\mathrm{mmol}$ ) was dilute with THF $(1 \mathrm{~mL})$ added drop wise at $0{ }^{\circ} \mathrm{C}$, stirred for $16 \mathrm{~h}$ at rt. Extracted with ethyl acetate (3 $\times 20 \mathrm{~mL}$ ) and dried over anhydrous $\mathrm{Na}_{2} \mathrm{SO}_{4}$ and concentrated in vacuum. The crude product was purified by column chromatography on silica gel (eluent: $0-50 \%$ DCM/EtOAC) to afford the product 1-(1- $((3,5-$ dichlorophenyl)sulfonyl)piperidin-4-yl)-2-(furan-2-yl)-1H-benzo[d]imidazole as an off white solid $(0.120 \mathrm{~g}$, 51.02\%); mp 189-192 ${ }^{\circ} \mathrm{C} ;{ }^{1} \mathrm{H} \mathrm{NMR}\left(400 \mathrm{MHz}\right.$, DMSO- $\left.d_{6}\right): \delta$ 1.92-2.00 (m, 2H), 2.34-2.42 (m, 2H), 2.79-2.85 (m, $2 \mathrm{H}), 3.97-4.00(\mathrm{~d}, J 12.0 \mathrm{~Hz}, 2 \mathrm{H}), 4.87(\mathrm{t}, J 24.4 \mathrm{~Hz}, 1 \mathrm{H}), 6.74(\mathrm{t}, J 4.8 \mathrm{~Hz}, 1 \mathrm{H}), 7.14(\mathrm{~d}, J 3.2 \mathrm{~Hz}, 1 \mathrm{H}), 7.22-7.27$ (m, 2H), 7.39-7.69 (m, 1H), 7.92 (d, J 1.6 Hz, 2H), $7.96(\mathrm{~d}, J 1.2 \mathrm{~Hz}, 1 \mathrm{H}), 8.17(\mathrm{~s}, 1 \mathrm{H}) ;{ }^{13} \mathrm{C}-\mathrm{NMR}(101 \mathrm{MHz}$, DMSO$\left.d_{6}\right): \delta 145.52,144.58,144.10,143.65,140.64,136.09,133.96,133.38,126.42,123.12,122.72,120.23,113.89$, 112.81, 112.47, 53.75, 45.93, 29.44; LC-MS, RT: $2.271 \mathrm{~min}, 100 \%$, גmax: 254 nm, MS: $m / z$ [M+1] 478.0; Anal. Calc. for $\mathrm{C}_{22} \mathrm{H}_{19} \mathrm{Cl}_{2} \mathrm{~N}_{3} \mathrm{O}_{3} \mathrm{~S}: \mathrm{C}, 55.47 ; \mathrm{H}, 4.02 ; \mathrm{N}, 8.82$; Found: $\mathrm{C}, 55.42 ; \mathrm{H}, 3.97 ; \mathrm{N}, 8.78 \%$.

\section{Acknowledgements}

The authors are thankful to the Shri M.M Patel Institute of Sciences and Research College Gandhinagar for providing laboratory facilities and to the Biotechnology Department of Pramukh Swami Science \& H. D. Patel Arts College, Kadi for providing biological activity.

\section{Supplementary Material}

Copies of ${ }^{1} \mathrm{H}$ NMR, ${ }^{13} \mathrm{C}$ NMR and LC-MS spectra of synthesized compounds are available in the Supplementary Material.

\section{References}

1. Heers, J.; Backx, L. J. J.; Mostmans, J. H.; Van Cutsem, J. J. Med. Chem. 1979, 22, 1003. https://doi.org/10.1021/jm00194a023

2. Hunkeler, W.; Mohler, H.; Pieri, L.; Polc, P.; Bonetti, E. P.; Cumin, R.; Schaffner, R.; Haefely, W. Nature 1981, 290, 514. 
3. Brimblecombe, R. W.; Duncan, W. A. M.; Durant, G. J.; Emmett, J. C.; Ganellin, C. R.; Parsons, M. E. J. Int. Med. Res. 1975, 3, 86.

https://doi.org/10.1177/030006057500300205

4. Tanigawara, Y.; Aoyama, N.; Kita, T.; Shirakawa, K.; Komada, F.; Kasuga, M.; Okumura, K. Clin. Pharmacol Ther. 1999, 66, 528.

https://doi.org/10.1016/S0009-9236(99)70017-2

5. Wauquier, A.; Van Den Broeck, W. A. E.; Verheyen, J. L.; Janssen, P. A. J. Eur. J. Pharmacol. 1978, 47, 367. https://doi.org/10.1016/0014-2999(78)90117-6

6. Lee, J. C.; Laydon, J. T.; McDonnell, P. C.; Gallagher, T. F.; Kumer, S.; Green, D.; McNulty, D.; Blumenthal, M. J.; Keys, J. R.; Vatter, S. W. L.; Strickler, J. E.; McLaughlin, M. M.; Siemens, I. R.; Fisher, S. M.; Livi, G. P.; White, J. R.; Adams, J. L.; Young, P. R. Nature 1994, 372, 739.

https://doi.org/10.1038/372739a0

7. De Laszlo, S. E.; Hacker, C.; Li, B.; Kim, D.; MacCoss, M.; Mantalo, N.; Pivnichnny, J. V.; Colwell, L.; Koch, G. E.; Cascieri, M. A.; Hagmenn, W. K. Bioorg. Med. Chem. Lett. 1999, 9, 641. https://doi.org/10.1016/S0960-894X(99)00081-5

8. Beaulieu, C.; Wang, Z.; Denis, D.; Greig, G.; Lamontagne, S.; O’Neill, G.; Slipetz, D.; Wang, J. Bioorg. Med. Chem. Lett. 2004, 14, 3195. https://doi.org/10.1016/j.bmcl.2004.04.005

9. Schmierer, R.; Mildenberger, H.; Buerstell, H. German Patent 1987, 361464; Chem. Abstr. 1988, 108, 37838.

10. Heeres, J.; Backx, L. J. J.; Mostmans, J. H.; Van Custem, J. J. Med. Chem. 1979, 22, 1003. https://doi.org/10.1021/jm00194a023

11. Antolini, M.; Bozzoli, A.; Ghiron, C.; Kennedy, G.; Rossi, T.; Ursini, A. Bioorg. Med. Chem. Lett. 1999, 9, 1023.

\section{https://doi.org/10.1016/S0960-894X(99)00112-2}

12. Wang, L.; Woods, K. W.; Li, Q.; Barr, K. J.; McCroskey, R. W.; Hannick, S. M.; Gherke, L.; Credo, R. B.; Hui, Y. H.; Marsh, K.; Warner, R.; Lee, J. Y.; Zielinsky-Mozong, N.; Frost, D.; Rosenberg, S. H.; Sham, H. L. J. Med. Chem. 2002, 45, 1697.

https://doi.org/10.1021/jm010523x

13. Grassi, A.; Ippen, J.; Bruno, M.; Thomas, G.; Bay, P. Eur. J. Pharmacol. 1991, 195, 251-259. https://doi.org/10.1016/0014-2999(91)90543-Y

14. Ozkay, Y.; Tunali, Y.; Karaca, H.; Isikdag, I. Eur. J. Med. Chem. 2010, 45, 3293-3298. https://doi.org/10.1016/j.ejmech.2010.04.012

15. The American Society of Health-System Pharmacists. Retrieved Aug 18, 2015.

16. Spasov, A. A.; Yozhitsa, I. N.; Bugaeva, L. I.; Anisimova, V. A. Pharm. Chem. J. 1999, 33, 232. https://doi.org/10.1007/BF02510042

17. Rückle, T.; Biamonte, M.; Grippi-Vallotton, T.; Arkinstall, S.; Cambet, Y.; Camps, M.; Gotteland, J. P. J. Med. Chem. 2004, 47, 6921-6934.

https://doi.org/10.1021/jm031112e

18. Wagner, E. C.; Millett, W. H. Org. Synth. 1943, 2, 65.

19. Grimmet, M. R.; Katritzky, A. R.; Rees, C. W. Comprehensive Heterocyclic Chemistry, Pergamon Press, Oxford, 1984, 5, 104-105.

20. Panda, S. S.; Malik, R.; Jain, S. C. Curr.Org. Chem. 2012, 16, 1905-1919.

https://doi.org/10.2174/138527212802651232 
21. Cee, V. J.; Downing, N. S. Tetrahedron Lett. 2006, 47, 3747-3750.

https://doi.org/10.1016/j.tetlet.2006.03.112

22. Sluiter, J.; Christoffers, J. Synlett 2009, 1, 63-66.

23. Bastug, G.; Eviolitte, C.; Markó; I. E. Org. Lett. 2012, 14, 3502-3505. https://doi.org/10.1021/ol301472a

24. Yan, Y.; Zhong, Q. F.; Zhao, N.; Liu, G. Mol. Divers. 2012, 16, 157-162. https://doi.org/10.1007/s11030-011-9343-0

25. Saha, P.; Ramana, T.; Purkait, N.; Ali, M. A.; Paul, R.; Punniyamurthy, T. J. Org. Chem. 2009, 74, 8719-8725. https://doi.org/10.1021/jo901813g

26. Wray, B. C.; Stambuli, J. P. Org. Lett. 2010, 12, 4576-4579. https://doi.org/10.1021/ol101899q

27. Oda, S; Shimizu, H.; Aoyama, Y.; Ueki, T. ; Shimizu, S.; Osato, H.; Takeuchi, Y. Org. Process Res. Dev. 2012, 16, 96-101.

https://doi.org/10.1021/op200251c

28. Yang, D.; Fokas, J.; Li, J.; Yu, L.; Baldino, C. M. Synthesis 2005, 1, 47-56. https://doi.org/10.1055/s-2004-834926

29. Kojima T, Mochizuki M, Takai T, Hoashi Y, Morimoto S, Seto M, Nakamura M, Kobayashi K, Sako Y, Tanaka M, Kanzaki N, Kosugi Y, Yano T, Aso K. Bioorg Med Chem. 2018, May 15;26(9), 2229-2250 https://doi.org/10.1016/i.bmc.2018.01.020 